Decay rates for radiative transitions in the Pr IV spectrum

This article has been downloaded from IOPscience. Please scroll down to see the full text article.

2013 J. Phys. B: At. Mol. Opt. Phys. 46145003

(http://iopscience.iop.org/0953-4075/46/14/145003)

View the table of contents for this issue, or go to the journal homepage for more

Download details:

IP Address: 109.89.160.191

The article was downloaded on 05/07/2013 at 12:32

Please note that terms and conditions apply. 


\title{
Decay rates for radiative transitions in the Pr IV spectrum
}

\author{
S Enzonga Yoca ${ }^{1}$ and P Quinet ${ }^{2,3}$ \\ ${ }^{1}$ Département de Physique, Faculté des Sciences et Techniques, Université Marien Ngouabi, \\ BP 69 Brazzaville, Congo \\ ${ }^{2}$ Astrophysique et Spectroscopie, Université de Mons-UMONS, B-7000 Mons, Belgium \\ ${ }^{3}$ IPNAS, Université de Liège, B-4000 Liège, Belgium \\ E-mail: quinet@umons.ac.be
}

Received 28 March 2013, in final form 5 June 2013

Published 5 July 2013

Online at stacks.iop.org/JPhysB/46/145003

\begin{abstract}
Transition probabilities and oscillator strengths for electric dipole radiation in triply ionized praseodymium are reported for the first time in this paper. They were computed using a semi-empirical relativistic Hartree-Fock approach including core-polarization effects. Due to the lack of experimental data in the Pr IV spectrum, the accuracy of our results is estimated and discussed on the basis of comparisons between calculations performed with a similar physical model and laboratory measurements previously published for the isoelectronic ion $\mathrm{Ce}^{2+}$. In view of their great interest in optical materials and nanophotonics, radiative rates for forbidden lines within the $4 \mathrm{f}^{2}$ ground-state configuration of $\operatorname{Pr}^{3+}$ were also calculated in our work.
\end{abstract}

\section{Introduction}

Because of their unique photophysical properties, in particular with respect to the generation and amplification of light, triply ionized lanthanide elements hold a special place in photonics. The luminescence of these ions in compounds has been widely studied and has found applications in many scientific domains such as the lighting industry, laser physics, optical telecommunications, molecular biology, medical diagnostics, etc (see e.g. Hemmilä 1995, Wybourne 2004, Hasegawa et al 2004, Dossing 2005).

This luminescence is the result of the competition of radiative and non-radiative relaxation processes of electronically excited states in lanthanide ions that are characterized by a multitude of energy levels particularly because of the unfilled $4 \mathrm{f}$ orbital. Understanding the light emission from triply ionized lanthanides therefore requires reliable information about their atomic structure and radiative parameters. However, our knowledge of the spectra corresponding to these ions is still very fragmentary, most of the experimentally identified energy levels being listed in the tables of the National Institute of Standards and Technology (NIST, see Kramida et al 2012) which are entirely based on the compilation due to Martin et al (1978). Recently, there has been a revival of the interest in triply ionized lanthanides, and several spectral analyses based on high-resolution vacuum ultraviolet observations have been completed. Among the spectra considered, let us mention, e.g. Nd IV (Wyart et al 2006, Wyart et al 2007), Tm IV (Meftah et al 2007) and Yb IV (Wyart et al 2001). Theoretical investigations of $4 \mathrm{f}^{k}$ groundstate configurations in $\operatorname{Pr} \operatorname{IV}(k=2)$ and $\operatorname{Nd} \operatorname{IV}(k=3)$ have also been carried out by Wyart et al (2008).

Concerning the radiative properties of triply ionized lanthanides, the investigations performed so far were focused on a rather limited number of electric dipole transitions in La IV (Biémont et al 2009), Ce IV (Migdalek and Baylis 1979, Migdalek and Wyrozumska 1987, Glushkov 1992, Zhang et al 2001c, Savukov et al 2003), Pr IV (Sen and Puri 1989, Stanek and Migdalek 2004, Wyart et al 2005), Nd IV, Pm IV, Sm IV, Eu IV, Gd IV (Dzuba et al 2003), Yb IV (Wyart et al 2001) and Lu IV (Loginov and Tuchkin 2001, Anisimova et al 2001). Very recently, theoretical emission rates and oscillator strengths were published by Dodson and Zia (2012) for some magnetic dipole and electric quadrupole transitions within the $4 \mathrm{f}^{k}(k=$ $1-13)$ configurations along the triply ionized lanthanide series from Ce IV to $\mathrm{Yb}$ IV.

In this work, the relativistic Hartree-Fock (HFR) approach, including core-polarization (CPOL) effects, has been used to estimate the radiative decay rates in $\mathrm{Pr}$ IV. The excellent agreement between experimental lifetime measurements and theoretical results obtained for the isoelectronic ion $\mathrm{Ce}^{2+}$ (see Biémont et al 2002) when using 
a similar HFR model allowed us to assess the reliability of the new set of transition probabilities. These calculations in Pr IV are an extension of our previous investigations of the triply charged lanthanide ions La IV (Biémont et al 2009), Ce IV (Zhang et al 2001c) and Yb IV (Wyart et al 2001). Due to their particular interest in optical physics and nanophotonics, the radiative parameters corresponding to forbidden transitions within the $4 \mathrm{f}^{2}$ ground-state configuration of Pr IV were also computed in our work and compared to available theoretical data.

\section{Level structure of Pr IV}

The ground state of $\operatorname{Pr}$ IV is $[\mathrm{Xe}] 4 \mathrm{f}^{2}{ }^{3} \mathrm{H}_{4}$. All of the term energies experimentally deduced so far are listed in the NIST compilation (Martin et al 1978) which contains 88 levels identified as belonging to the $4 \mathrm{f}^{2}, 4 \mathrm{f} 5 \mathrm{f}, 4 \mathrm{f} 6 \mathrm{p}, 5 \mathrm{~d}^{2}$ even configurations and to the $4 \mathrm{f} 5 \mathrm{~d}, 4 \mathrm{f} 6 \mathrm{~d}, 4 \mathrm{f} 6 \mathrm{~s}, 4 \mathrm{f} 7 \mathrm{~s}, 5 \mathrm{~d} 6 \mathrm{p}$ odd configurations. This critical compilation was essentially based on the works of Sugar $(1965,1971 \mathrm{a})$ and Crosswhite et al (1965) who observed the Pr IV spectrum emitted by sliding-spark discharges and reported line lists covering the wavelength region from 69.1 to $302.1 \mathrm{~nm}$. The level compositions in the $4 \mathrm{f}^{2}$ configuration were taken from the theoretical analysis of Goldschmidt (1968) later refined to include additional magnetic interactions by Goldschmidt et al (1968) and Pasternak (1970). Theoretical percentages for levels of the 4f6s, 4f5d, 4f6d, 4f6p and 4f5f configurations were taken from Sugar $(1965,1971$ a) supplemented by some of his unpublished results (Sugar 1971b).

More recently, a theoretical study of the $4 \mathrm{f}^{2}$ ground-state configuration in Pr IV was published by Wyart et al (2008) who performed a parametric fit of level energies, taking into account the Coulomb and spin-dependent interactions beyond the first order of perturbation. In this study, an excellent agreement between theoretical and experimental energies was observed, the root-mean square deviation being found equal to only $1.29 \mathrm{~cm}^{-1}$ for the 12 known levels within the $4 \mathrm{f}^{2}$ configuration. This excellent agreement allowed the authors to predict an energy value of $48044.66 \mathrm{~cm}^{-1}$ with a good accuracy for the one remaining unknown level in $4 \mathrm{f}^{2}$, i.e. ${ }^{1} \mathrm{~S}_{0}$.

\section{Radiative parameter calculations}

$\mathrm{Pr}^{3+}$ ion is a Ba-like atomic system with two valence electrons surrounding a Xe-like core. As a consequence, intravalence and core-valence interactions should be both taken into account for calculating the atomic structure. In addition, relativistic effects must normally play an important role. A method which has appeared as a suitable compromise between a gratifying accuracy of the results (tested by comparison with accurate laser lifetime measurements), the moderate complexity of the codes used and the ability to obtain many new results in a limited CPU time, is the pseudo-relativistic Hartree-Fock (HFR) technique as described by Cowan (1981), but modified by us for the inclusion of CPOL effects. In this approach (HFR + CPOL), most of the intravalence correlation is represented within a configuration interaction scheme, while the core-valence correlation is described by a CPOL model potential and a correction to the dipole operator depending upon two parameters, i.e. the dipole polarizability of the ionic core, $\alpha_{d}$, and the cut-off radius, $r_{c}$ (for details see e.g. Quinet et al 1999). Although based on the Schrödinger equation, this method takes the most important relativistic effects, such as the mass-velocity contribution and the Darwin term, into account.

The physical HFR + CPOL model used for Pr IV was exactly the same as the one described in our previous paper related to the isoelectronic ion Ce III (Biémont et al 2002). In this model, the intravalence correlation was explicitly retained among the following configurations : $4 \mathrm{f}^{2}+4 \mathrm{f} n \mathrm{p}(n=6-7)+$ $5 \mathrm{~d}^{2}+5 \mathrm{~d} n \mathrm{~s}(n=6-7)+6 \mathrm{~s}^{2}+5 \mathrm{~d} 6 \mathrm{~d}+4 \mathrm{f} n \mathrm{f}(n=5-7)+6 \mathrm{p}^{2}$ for the even parity and $4 \mathrm{f} n \mathrm{~d}(n=5-7)+4 \mathrm{f} n \mathrm{~s}(n=6-8)+$ $5 \mathrm{~d} 6 \mathrm{p}+4 \mathrm{f} n \mathrm{~g}(n=5-6)+6 \mathrm{~s} 6 \mathrm{p}$ for the odd parity. CPOL effects were included using the dipole polarizability, $\alpha_{d}$, equal to 5.40 $\mathrm{a}_{0}^{3}$, as tabulated by Fraga et al (1976) for the Xe-like ionic core $\mathrm{Pr}^{5+}$, while the cut-off radius, $r_{c}$, was chosen to be equal to $1.60 \mathrm{a}_{0}$ which corresponds to the expected value of $r$ for the outermost core orbital $\left(5 p^{6}\right)$ as computed with Cowan's codes. However, as already mentioned in many previous papers on lowly ionized lanthanides (see e.g. Biémont et al 2001a, Li et al 2001), the inadequacy of the analytical polarization corrections to the dipole operator as introduced in the HFR + CPOL model for transitions involving the $4 \mathrm{f}$ electrons imposes the consideration of a scaling factor to the $\langle 4 \mathrm{f}|r| n \mathrm{~d}\rangle$ and $\langle 4 \mathrm{f}|r| n \mathrm{~g}\rangle$ dipole operators in order to compensate for the sudden collapse of the $4 \mathrm{f}$ orbital inside the Xe-like core. In this work, those operators were scaled down by the same factor as the one used in the case of Ce III, i.e. 0.80 (Biémont et al 2002). A similar approach was already successfully considered in many different ions of the lanthanide series, an excellent agreement (within a few per cent) between theoretical and experimental lifetimes having been found in Ce II (Zhang et al 2001b), Pr III (Biémont et al 2001a), Tm III (Li et al 2001), Er III (Biémont et al 2001b), Yb III (Zhang et al 2001a) and Yb IV (Wyart et al 2001).

Using a least-squares fitting procedure (Cowan 1981), average energies, Slater integrals, spin-orbit and effective interaction parameters were adjusted to obtain the best agreement between the calculated and the experimental energy levels taken from the NIST tables (Martin et al 1978). All the values listed in this compilation were used in the fit process if we except the two 5d6p levels at 195917.0 and $202487.0 \mathrm{~cm}^{-1}$. Note that the accurate predicted value obtained by Wyart et al (2008) for the ${ }^{1} \mathrm{~S}_{0}$ level of $4 \mathrm{f}^{2}(E=$ $48044.66 \mathrm{~cm}^{-1}$ ) was also used to obtain a complete set of parameters in this configuration. The radial parameters adopted in this work are given in table 1 (even parity) and table 2 (odd parity), while the energy levels computed with those parameters are compared with available experimental values in tables 3 and 4, respectively. As seen from these tables, the average deviations were found to be equal to $34 \mathrm{~cm}^{-1}$ for even-parity levels and to $95 \mathrm{~cm}^{-1}$ for odd-parity levels (which means $1 / 6000$ and $1 / 2100$ of the energy ranges, respectively). We also note that, for the $4 \mathrm{f} 6 \mathrm{p}$ configuration, our parameters $\mathrm{G}^{2}(4 \mathrm{f}, 6 \mathrm{p})$ and $\mathrm{G}^{4}(4 \mathrm{f}, 6 \mathrm{p})$ differ by $20 \%$ from those deduced 
Table 1. Adopted radial parameters (in $\mathrm{cm}^{-1}$ ) for even-parity configurations of Pr IV.

\begin{tabular}{|c|c|c|c|c|c|}
\hline Configuration & Parameter & HFR & Fitted & Fitted/HFR & Note \\
\hline \multirow[t]{8}{*}{$4 f^{2}$} & $\mathrm{E}_{a v}$ & 11981.0 & 10400.3 & & \\
\hline & $\mathrm{F}^{2}(4 \mathrm{f}, 4 \mathrm{f})$ & 98646.2 & 73738.1 & 0.75 & \\
\hline & $F^{4}(4 f, 4 f)$ & 61885.0 & 54930.9 & 0.89 & \\
\hline & $\mathrm{F}^{6}(4 \mathrm{f}, 4 \mathrm{f})$ & 44517.7 & 36754.6 & 0.83 & \\
\hline & $\alpha$ & 0.0 & 12.5 & & \\
\hline & $\beta$ & 0.0 & -293.7 & & \\
\hline & $\gamma$ & 0.0 & 514.1 & & \\
\hline & $\zeta_{4 f}$ & 826.8 & 757.6 & 0.92 & \\
\hline \multirow[t]{6}{*}{$4 f 6 p$} & $\mathrm{E}_{a v}$ & 138401.5 & 142460.0 & & \\
\hline & $\zeta_{4 \mathrm{f}}$ & 919.6 & 856.1 & 0.93 & \\
\hline & $\zeta_{6 \mathrm{p}}$ & 2792.8 & 3221.7 & 1.15 & \\
\hline & $\mathrm{F}^{2}(4 \mathrm{f}, 6 \mathrm{p})$ & 10058.8 & 8469.4 & 0.84 & \\
\hline & $\mathrm{G}^{2}(4 \mathrm{f}, 6 \mathrm{p})$ & 2542.6 & 3352.5 & 1.32 & \\
\hline & $G^{4}(4 f, 6 p)$ & 2340.0 & 3037.5 & 1.30 & \\
\hline \multirow[t]{6}{*}{$5 d^{2}$} & $\mathrm{E}_{a v}$ & 162046.9 & 148622.1 & & \\
\hline & $\mathrm{F}^{2}(5 \mathrm{~d}, 5 \mathrm{~d})$ & 49823.4 & 44841.1 & 0.90 & Fixed $^{\mathrm{a}}$ \\
\hline & $\mathrm{F}^{4}(5 \mathrm{~d}, 5 \mathrm{~d})$ & 33495.6 & 30146.0 & 0.90 & Fixed $^{\mathrm{a}}$ \\
\hline & $\alpha$ & 0.0 & 0.0 & & \\
\hline & $\beta$ & 0.0 & 0.0 & & \\
\hline & $\zeta_{5 \mathrm{~d}}$ & 1220.9 & 1471.7 & 1.16 & \\
\hline \multirow[t]{10}{*}{$4 \mathrm{f} 5 \mathrm{f}$} & $\mathrm{E}_{a v}$ & 199462.9 & 203100.4 & & \\
\hline & $\zeta_{4 \mathrm{f}}$ & 920.9 & 864.9 & 0.94 & \\
\hline & $\zeta_{5 \mathrm{f}}$ & 40.4 & 40.7 & 1.01 & \\
\hline & $F^{2}(4 f, 5 f)$ & 7603.8 & 5960.0 & 0.78 & \\
\hline & $F^{4}(4 f, 5 f)$ & 3137.6 & 4081.3 & 1.30 & \\
\hline & $F^{6}(4 f, 5 f)$ & 1996.3 & 3270.0 & 1.63 & \\
\hline & $G^{0}(4 f, 5 f)$ & 4509.7 & 1837.2 & 0.41 & \\
\hline & $G^{2}(4 f, 5 f)$ & 3352.1 & 3236.3 & 0.97 & \\
\hline & $G^{4}(4 f, 5 f)$ & 2326.4 & 3191.4 & 1.37 & \\
\hline & $G^{6}(4 f, 5 f)$ & 1734.6 & 1747.2 & 1.01 & \\
\hline
\end{tabular}

${ }^{a}$ Fixed parameter value in the fitting process.

Table 2. Adopted radial parameters (in $\mathrm{cm}^{-1}$ ) for odd-parity configurations of Pr IV.

\begin{tabular}{|c|c|c|c|c|c|}
\hline Configuration & Parameter & HFR & Fitted & Fitted/HFR & Note \\
\hline \multirow[t]{8}{*}{$4 f 5 d$} & $E_{a v}$ & 63250.0 & 67309.7 & & \\
\hline & $\zeta_{4 \mathrm{f}}$ & 911.6 & 922.1 & 1.01 & \\
\hline & $\zeta_{5 d}$ & 1074.1 & 1012.2 & 0.94 & \\
\hline & $F^{2}(4 f, 5 d)$ & 30877.1 & 22249.0 & 0.72 & \\
\hline & $F^{4}(4 f, 5 d)$ & 15423.3 & 17126.6 & 1.11 & \\
\hline & $G^{1}(4 f, 5 d)$ & 13563.4 & 10483.3 & 0.77 & \\
\hline & $G^{3}(4 f, 5 d)$ & 11498.8 & 12503.4 & 1.09 & \\
\hline & $G^{5}(4 f, 5 d)$ & 8912.6 & 9067.9 & 1.02 & \\
\hline \multirow[t]{8}{*}{$4 \mathrm{f} 6 \mathrm{~d}$} & $E_{a v}$ & 191463.7 & 196063.7 & & \\
\hline & $\zeta_{4 \mathrm{f}}$ & 920.6 & 880.8 & 0.96 & \\
\hline & $\zeta_{6 \mathrm{~d}}$ & 253.5 & 238.8 & 0.94 & \\
\hline & $F^{2}(4 f, 6 d)$ & 7001.3 & 4817.9 & 0.69 & $\mathrm{r}^{\mathrm{a}}$ \\
\hline & $F^{4}(4 f, 6 d)$ & 3080.6 & 2119.9 & 0.69 & $\mathrm{r}^{\mathrm{a}}$ \\
\hline & $G^{1}(4 f, 6 d)$ & 2247.3 & 2173.3 & 0.97 & $\mathrm{r} 2^{\mathrm{b}}$ \\
\hline & $G^{3}(4 f, 6 d)$ & 2147.1 & 2076.4 & 0.97 & $\mathrm{r} 2^{\mathrm{b}}$ \\
\hline & $G^{5}(4 f, 6 d)$ & 1733.8 & 1676.6 & 0.97 & $\mathrm{r} 2^{\mathrm{b}}$ \\
\hline \multirow[t]{3}{*}{$4 f 6 s$} & $E_{a v}$ & 99087.4 & 102304.9 & & \\
\hline & $\zeta_{4 \mathrm{f}}$ & 918.8 & 860.3 & 0.94 & \\
\hline & $\mathrm{G}^{3}(4 \mathrm{f}, 6 \mathrm{~s})$ & 3396.2 & 2827.1 & 0.83 & \\
\hline \multirow[t]{3}{*}{$4 \mathrm{f} 7 \mathrm{~s}$} & $E_{a v}$ & 196972.3 & 201525.9 & & \\
\hline & $\zeta_{4 \mathrm{f}}$ & 921.6 & 921.6 & 1.00 & Fixed $^{\mathrm{c}}$ \\
\hline & $\mathrm{G}^{3}(4 \mathrm{f}, 7 \mathrm{~s})$ & 1186.9 & 1068.2 & 0.90 & Fixed $^{c}$ \\
\hline
\end{tabular}

${ }^{a}$ Fixed ratio between these parameters in the fitting process.

${ }^{\mathrm{b}}$ Fixed ratio between these parameters in the fitting process.

${ }^{\mathrm{c}}$ Fixed parameter value in the fitting process. 
Table 3. Experimental and calculated even-parity energy levels in Pr IV.

\begin{tabular}{|c|c|c|c|c|}
\hline$E_{\exp }{ }^{\mathrm{a}}\left(\mathrm{cm}^{-1}\right)$ & $E_{\text {calc }}\left(\mathrm{cm}^{-1}\right)$ & $\Delta E\left(\mathrm{~cm}^{-1}\right)$ & $J$ & $L S$ composition $^{\mathrm{b}}$ \\
\hline 0.00 & 24 & -24 & 4 & $97 \% 4 \mathrm{f}^{2}{ }^{3} \mathrm{H}$ \\
\hline 2152.09 & 2147 & 5 & 5 & $100 \% 4 \mathrm{f}^{2}{ }^{3} \mathrm{H}$ \\
\hline 4389.09 & 4371 & 18 & 6 & $100 \% 4 \mathrm{f}^{2}{ }^{3} \mathrm{H}$ \\
\hline 4996.61 & 4990 & 7 & 2 & $98 \% 4 \mathrm{f}^{2}{ }^{3} \mathrm{~F}$ \\
\hline 6415.24 & 6405 & 10 & 3 & $100 \% 4 \mathrm{f}^{2}{ }^{3} \mathrm{~F}$ \\
\hline 6854.75 & 6890 & -35 & 4 & $65 \% 4 \mathrm{f}^{2}{ }^{3} \mathrm{~F}+33 \% 4 \mathrm{f}^{2}{ }^{1} \mathrm{G}$ \\
\hline 9921.24 & 9902 & 19 & 4 & $64 \% 4 \mathrm{f}^{2}{ }^{1} \mathrm{G}+34 \% 4 \mathrm{f}^{2}{ }^{3} \mathrm{~F}$ \\
\hline 17334.39 & 17329 & 5 & 2 & $90 \% 4 \mathrm{f}^{2}{ }^{1} \mathrm{D}+8 \% 4 \mathrm{f}^{2}{ }^{3} \mathrm{P}$ \\
\hline 21389.81 & 21352 & 38 & 0 & $99 \% 4 \mathrm{f}^{2}{ }^{3} \mathrm{P}$ \\
\hline 22007.46 & 21991 & 16 & 1 & $100 \% 4 \mathrm{f}^{2}{ }^{3} \mathrm{P}$ \\
\hline 22211.54 & 22212 & 0 & 6 & $100 \% 4 \mathrm{f}^{2}{ }^{1} \mathrm{I}$ \\
\hline \multirow[t]{2}{*}{23160.61} & 23220 & -59 & 2 & $92 \% 4 \mathrm{f}^{2}{ }^{3} \mathrm{P}+8 \% 4 \mathrm{f}^{21} \mathrm{~d}$ \\
\hline & 48045 & 0 & 0 & $97 \% 4 \mathrm{f}^{21} \mathrm{~S}$ \\
\hline 136850.85 & 136941 & -90 & 3 & $61 \% 4 f 6 p{ }^{3} G+28 \% 4 f 6 p{ }^{1} F+10 \% 4 f 6 p{ }^{3} F$ \\
\hline 137175.04 & 137104 & 71 & 2 & $65 \% 4 f 6 p^{3} F+15 \% 4 f 6 p{ }^{1} D+11 \% 4 f 6 p^{3} D$ \\
\hline 139711.8 & 139706 & 6 & 2 & $84 \% 5 \mathrm{~d}^{2}{ }^{3} \mathrm{~F}+5 \% 4 \mathrm{f} 6 \mathrm{p}^{3} \mathrm{D}$ \\
\hline 139875.31 & 139803 & 72 & 3 & $54 \% 4 f 6 p^{3} F+26 \% 4 f 6 p^{3} D+13 \% 4 f 6 p{ }^{1} F$ \\
\hline 140225.92 & 140270 & -44 & 4 & $43 \% 4 f 6 p^{3} G+40 \% 4 f 6 p^{3} F+15 \% 4 f 6 p{ }^{1} G$ \\
\hline 141254.01 & 141221 & 33 & 3 & $35 \% 4 f 6 p^{3} G+31 \% 4 f 6 p{ }^{1} F+14 \% 4 f 6 p^{3} F$ \\
\hline 142331.59 & 142438 & -107 & 2 & $53 \% 4 f 6 p^{3} D+30 \% 4 f 6 p^{3} F+15 \% 4 f 6 p^{1} D$ \\
\hline \multirow[t]{2}{*}{142565.94} & 142507 & 59 & 4 & $56 \% 4 f 6 p^{3} G+21 \% 4 f 6 p^{3} F+19 \%$ \\
\hline & 142609 & & 3 & $81 \% 5 d^{2}{ }^{3} \mathrm{~F}+11 \% 4 \mathrm{f} 6 \mathrm{p}^{3} \mathrm{~F}$ \\
\hline 142997.32 & 142963 & 34 & 1 & $99 \% 4 f 6 p^{3} \mathrm{D}$ \\
\hline 144925.33 & 144818 & 107 & 4 & $48 \% 4 \mathrm{f} 6 \mathrm{p}{ }^{1} \mathrm{G}+41 \% 5 \mathrm{~d}^{2}{ }^{3} \mathrm{~F}+10 \% 4 \mathrm{f} 6 \mathrm{p}^{3} \mathrm{~F}$ \\
\hline 144943.27 & 144984 & -41 & 3 & $61 \% 4 f 6 p{ }^{3} D+25 \% 4 f 6 p{ }^{1} F+12 \% 4 f 6 p{ }^{3} F$ \\
\hline 145281.20 & 145214 & 67 & 5 & $100 \% 4 \mathrm{f} 6 \mathrm{p}^{3} \mathrm{G}$ \\
\hline 145362.7 & 145505 & -143 & 4 & $50 \% 5 d^{2}{ }^{3} \mathrm{~F}+28 \% 4 \mathrm{f} 6 \mathrm{p}^{3} \mathrm{~F}+16 \% 4 \mathrm{f} 6 \mathrm{p}^{1} \mathrm{G}$ \\
\hline \multirow[t]{7}{*}{146577.13} & 146604 & -27 & 2 & $65 \% 4 f 6 p{ }^{1} D+31 \% 4 f 6 p^{3} D$ \\
\hline & 149145 & & 2 & $65 \% 5 \mathrm{~d}^{2}{ }^{1} \mathrm{D}+30 \% 5 \mathrm{~d}^{2}{ }^{3} \mathrm{P}$ \\
\hline & 149505 & & 0 & $97 \% 5 \mathrm{~d}^{2}{ }^{3} \mathrm{P}$ \\
\hline & 150806 & & 1 & $99 \% 5 \mathrm{~d}^{2}{ }^{3} \mathrm{P}$ \\
\hline & 153641 & & 2 & $69 \% 5 \mathrm{~d}^{2}{ }^{3} \mathrm{P}+29 \% 5 \mathrm{~d}^{2}{ }^{1} \mathrm{D}$ \\
\hline & 155161 & & 4 & $95 \% 5 \mathrm{~d}^{2}{ }^{1} \mathrm{G}$ \\
\hline & 172271 & & 0 & $93 \% 5 d^{21} \mathrm{~S}$ \\
\hline 199191.0 & 199185 & 6 & 3 & $69 \% 4 f 5 f^{3} \mathrm{G}+27 \% 4 \mathrm{f} 5 \mathrm{f}^{1} \mathrm{~F}$ \\
\hline 199595.8 & 199635 & -40 & 5 & $53 \% 4 \mathrm{f} 5 \mathrm{f}^{3} \mathrm{I}+38 \% 4 \mathrm{f} 5 \mathrm{f}{ }^{1} \mathrm{H}+6 \% 4 \mathrm{f} 5 \mathrm{f}^{3} \mathrm{G}$ \\
\hline 199815.5 & 199729 & 86 & 1 & $57 \% 4 \mathrm{f} 5 \mathrm{f}^{3} \mathrm{D}+36 \% 4 \mathrm{f} 5 \mathrm{f}{ }^{1} \mathrm{P}$ \\
\hline 200202.6 & 200191 & 11 & 4 & $87 \% 4 f 5 f^{3} G+5 \% 4 f 5 f^{3} H$ \\
\hline 200235.1 & 200245 & -10 & 5 & $42 \% 4 \mathrm{f} 5 \mathrm{f}^{3} \mathrm{I}+27 \% 4 \mathrm{f} 5 \mathrm{f}^{1} \mathrm{H}+17 \% 4 \mathrm{f} 5 \mathrm{f}^{3} \mathrm{G}$ \\
\hline 200417.0 & 200409 & 8 & 3 & $33 \% 4 \mathrm{f} 5 \mathrm{f}^{3} \mathrm{D}+31 \% 4 \mathrm{f} 5 \mathrm{f}{ }^{1} \mathrm{~F}+21 \% 4 \mathrm{f} 5 \mathrm{f}^{3} \mathrm{G}$ \\
\hline 200697.1 & 200722 & -25 & 2 & $85 \% 4 f 5 f{ }^{3} D+6 \% 4 f 5 f{ }^{3} F$ \\
\hline 200893.8 & 200936 & -42 & 6 & $89 \% 4 \mathrm{f} 5 \mathrm{f}^{3} \mathrm{I}+7 \% 4 \mathrm{f} 5 \mathrm{f}^{1} \mathrm{I}$ \\
\hline 201365.2 & 201358 & 7 & 1 & $43 \% 4 \mathrm{f} 5 \mathrm{f}^{3} \mathrm{~S}+22 \% 4 \mathrm{f} 5 \mathrm{f}^{3} \mathrm{D}+21 \% 4 \mathrm{f} 5 \mathrm{f}^{1} \mathrm{P}$ \\
\hline 201985.3 & 202024 & -39 & 4 & $82 \% 4 \mathrm{f} 5 \mathrm{f}^{3} \mathrm{H}+15 \% 4 \mathrm{f} 5 \mathrm{f}^{1} \mathrm{G}$ \\
\hline \multirow[t]{2}{*}{202327.9} & 202311 & 17 & 5 & $72 \% 4 \mathrm{f} 5 \mathrm{f}^{3} \mathrm{G}+25 \% 4 \mathrm{f} 5 \mathrm{f}^{1} \mathrm{H}$ \\
\hline & 202441 & & 1 & $83 \% 5 \mathrm{~d} 6 \mathrm{~s}^{3} \mathrm{D}+8 \% 4 \mathrm{f} 5 \mathrm{f}^{3} \mathrm{~S}$ \\
\hline 202614.7 & 202593 & 21 & 3 & $54 \% 4 \mathrm{f} 5 \mathrm{f}^{3} \mathrm{D}+38 \% 4 \mathrm{f} 5 \mathrm{f}{ }^{1} \mathrm{~F}+6 \% 4 \mathrm{f} 5 \mathrm{f}^{3} \mathrm{G}$ \\
\hline 202819.0 & 202775 & 44 & 2 & $63 \% 4 \mathrm{f} 5 \mathrm{f}^{3} \mathrm{~F}+20 \% 4 \mathrm{f} 5 \mathrm{f}^{1} \mathrm{D}+11 \% 5 \mathrm{~d} 6 \mathrm{~s}^{3} \mathrm{D}$ \\
\hline 202948.9 & 202997 & -48 & 7 & $100 \% 4 \mathrm{f} 5 \mathrm{f}^{3} \mathrm{I}$ \\
\hline \multirow[t]{2}{*}{203265.4} & 203254 & 11 & 1 & $40 \% 4 f 5 f{ }^{1} \mathrm{P}+38 \% 4 \mathrm{f} 5 \mathrm{f}{ }^{3} \mathrm{~S}+16 \% 4 \mathrm{f} 5 \mathrm{f}{ }^{3} \mathrm{D}$ \\
\hline & 203394 & & 2 & $74 \% 5 \mathrm{~d} 6 \mathrm{~s}{ }^{3} \mathrm{D}+9 \% 4 \mathrm{f} 5 \mathrm{f}^{3} \mathrm{~F}+5 \% 4 \mathrm{f} 5 \mathrm{f}^{3} \mathrm{D}$ \\
\hline 204032.1 & 204054 & -22 & 5 & $84 \% 4 \mathrm{f} 5 \mathrm{f}^{3} \mathrm{H}+9 \% 4 \mathrm{f} 5 \mathrm{f}{ }^{1} \mathrm{H}$ \\
\hline 204557.0 & 204554 & 3 & 3 & $85 \% 4 \mathrm{f} 5 \mathrm{f}^{3} \mathrm{~F}+7 \% 4 \mathrm{f} 5 \mathrm{f}^{3} \mathrm{D}$ \\
\hline 204541.7 & 204565 & -23 & 4 & $41 \% 4 f 5 f{ }^{3} F+36 \% 4 f 5 f{ }^{1} G+12 \% 4 f 5 f{ }^{3} G$ \\
\hline \multirow[t]{2}{*}{204815.6} & 204801 & 15 & 6 & $90 \% 4 \mathrm{f} 5 \mathrm{f}^{3} \mathrm{H}+5 \% 4 \mathrm{f} 5 \mathrm{f}^{3} \mathrm{I}$ \\
\hline & 205173 & & 0 & $88 \% 4 \mathrm{f} 5 \mathrm{f}^{3} \mathrm{P}+9 \% 4 \mathrm{f} 5 \mathrm{f}{ }^{1} \mathrm{~S}$ \\
\hline \multirow[t]{2}{*}{205541.6} & 205535 & 6 & 2 & $48 \% 4 \mathrm{f} 5 \mathrm{f}{ }^{1} \mathrm{D}+20 \% 4 \mathrm{f} 5 \mathrm{f}{ }^{3} \mathrm{P}+20 \% 4 \mathrm{f} 5 \mathrm{f}^{3} \mathrm{~F}$ \\
\hline & 205634 & & 3 & $91 \% 5 \mathrm{~d} 6 \mathrm{~s}^{3} \mathrm{D}$ \\
\hline 205748.3 & 205700 & 48 & 4 & $51 \% 4 f 5 f^{3} F+45 \% 4 f 5 f^{1} G$ \\
\hline 206299.4 & 206319 & -20 & 6 & $87 \% 4 \mathrm{f} 5 \mathrm{f}^{1} \mathrm{I}+7 \% 4 \mathrm{f} 5 \mathrm{f}^{3} \mathrm{H}+5 \% 4 \mathrm{f} 5 \mathrm{f}^{3} \mathrm{I}$ \\
\hline 206369.4 & 206376 & -7 & 1 & $89 \% 4 \mathrm{f} 5 \mathrm{f}^{3} \mathrm{P}+7 \% 4 \mathrm{f} 5 \mathrm{f}^{3} \mathrm{~S}$ \\
\hline 207046.3 & 207044 & 2 & 2 & $70 \% 4 f 5 f^{3} \mathrm{P}+22 \% 4 \mathrm{f} 5 \mathrm{f}^{1} \mathrm{D}$ \\
\hline
\end{tabular}

${ }^{\text {a }}$ From Martin et al (1978).

${ }^{\mathrm{b}}$ Composition in \%. Only the first three main components larger than $5 \%$ are given. 
Table 4. Experimental and calculated odd-parity energy levels in Pr IV.

\begin{tabular}{|c|c|c|c|c|}
\hline $\begin{array}{l}E_{\exp { }^{\mathrm{a}}}\left(\mathrm{cm}^{-1}\right)\end{array}$ & $\begin{array}{l}E_{\text {calc }} \\
\left(\mathrm{cm}^{-1}\right)\end{array}$ & $\begin{array}{l}\Delta E \\
\left(\mathrm{~cm}^{-1}\right)\end{array}$ & $J$ & $L S$ composition $^{\mathrm{b}}$ \\
\hline 61170.95 & 61324 & -153 & 4 & $51 \% 4 \mathrm{f} 5 \mathrm{~d}^{1} \mathrm{G}+43 \% 4 \mathrm{f} 5 \mathrm{~d}^{3} \mathrm{H}$ \\
\hline 61457.48 & 61662 & -204 & 2 & $76 \% 4 f 5 d^{3} F+23 \% 4 f 5 d^{1} D$ \\
\hline 63355.94 & 63187 & 169 & 3 & $87 \% 4 f 5 d^{3} G+7 \% 4 f 5 d^{3} F+6 \% 4 f 5 d^{1} F$ \\
\hline 63580.59 & 63515 & 65 & 4 & $56 \% 4 f 5 d^{3} \mathrm{H}+33 \% 4 \mathrm{f} 5 \mathrm{~d}^{1} \mathrm{G}+10 \% 4 \mathrm{f} 5 \mathrm{~d}^{3} \mathrm{~F}$ \\
\hline 64123.54 & 64086 & 38 & 3 & $92 \% 4 f 5 d^{3} F+8 \% 4 f 5 d^{3} G$ \\
\hline 65239.39 & 64987 & 252 & 5 & $100 \% 4 \mathrm{f} 5 \mathrm{~d}^{3} \mathrm{H}$ \\
\hline 65639.95 & 65535 & 105 & 4 & $91 \% 4 f 5 d^{3} G+9 \% 4 f 5 d^{3} F$ \\
\hline 65321.67 & 65701 & -379 & 2 & $61 \% 4 f 5 d^{1} D+24 \% 4 f 5 d^{3} F+12 \% 4 f 5 d^{3} D$ \\
\hline 66518.01 & 66644 & -126 & 4 & $77 \% 4 f 5 d^{3} F+16 \% 4 f 5 d^{1} G+6 \% 4 f 5 d^{3} G$ \\
\hline 66967.72 & 66819 & 148 & 1 & $95 \% 4 \mathrm{f} 5 \mathrm{~d}^{3} \mathrm{D}$ \\
\hline 67899.32 & 67763 & 136 & 5 & $98 \% 4 f 5 d^{3} G$ \\
\hline 68077.83 & 67878 & 200 & 6 & $100 \% 4 \mathrm{f} 5 \mathrm{~d}^{3} \mathrm{H}$ \\
\hline 68411.51 & 68487 & -76 & 2 & $87 \% 4 f 5 d^{3} D+10 \% 4 f 5 d^{1} D$ \\
\hline 68495.57 & 68513 & -18 & 3 & $69 \% 4 \mathrm{f} 5 \mathrm{~d}^{3} \mathrm{D}+28 \% 4 \mathrm{f} 5 \mathrm{~d}^{1} \mathrm{~F}$ \\
\hline 70755.33 & 70710 & 45 & 1 & $93 \% 4 \mathrm{f} 5 \mathrm{~d}^{3} \mathrm{P}+5 \% 4 \mathrm{f} 5 \mathrm{~d}^{1} \mathrm{P}$ \\
\hline 70842.93 & 70771 & 72 & 0 & $100 \% 4 \mathrm{f} 5 \mathrm{~d}^{3} \mathrm{P}$ \\
\hline 71724.77 & 71785 & -61 & 3 & $66 \% 4 f 5 d^{1} F+31 \% 4 f 5 d^{3} D$ \\
\hline 72185.10 & 72385 & -200 & 2 & $94 \% 4 \mathrm{f} 5 \mathrm{~d}^{3} \mathrm{P}+5 \% 4 \mathrm{f} 5 \mathrm{~d}^{1} \mathrm{D}$ \\
\hline 75265.66 & 75315 & -49 & 5 & $98 \% 4 \mathrm{f} 5 \mathrm{~d}^{1} \mathrm{H}$ \\
\hline 78776.38 & 78743 & 33 & 1 & $91 \% 4 \mathrm{f} 5 \mathrm{~d}{ }^{1} \mathrm{P}+6 \% 4 \mathrm{f} 5 \mathrm{~d}^{3} \mathrm{P}$ \\
\hline 100258.48 & 100258 & 0 & 2 & $100 \% 4 \mathrm{f} 6 \mathrm{~s}^{3} \mathrm{~F}$ \\
\hline 100543.85 & 100545 & -1 & 3 & $69 \% 4 \mathrm{f} 6 \mathrm{~s}{ }^{3} \mathrm{~F}+31 \% 4 \mathrm{f} 6 \mathrm{~s}{ }^{1} \mathrm{~F}$ \\
\hline 103271.38 & 103272 & -1 & 4 & $100 \% 4 \mathrm{f} 6 \mathrm{~s}^{3} \mathrm{~F}$ \\
\hline 103753.75 & 103753 & 1 & 3 & $69 \% 4 \mathrm{f} 6 \mathrm{~s}{ }^{1} \mathrm{~F}+31 \% 4 \mathrm{f} 6 \mathrm{~s}^{3} \mathrm{~F}$ \\
\hline 193330.5 & 193403 & -73 & 2 & $80 \% 4 f 6 d^{3} F+19 \% 4 f 6 d^{1} D$ \\
\hline 193601.8 & 193457 & 145 & 4 & $63 \% 4 f 6 d^{3} H+30 \% 4 f 6 d^{1} G$ \\
\hline 193805.1 & 193853 & -48 & 3 & $84 \% 4 f 6 d^{3} G+14 \% 4 f 6 d^{1} F$ \\
\hline \multirow[t]{4}{*}{194022.2} & 194056 & -34 & 4 & $36 \% 4 f 6 d^{3} G+24 \% 4 f 6 d^{1} G+31 \% 4 f 6 d^{3} H$ \\
\hline & 194166 & & 3 & $71 \% 4 f 6 d^{3} F+16 \% 4 f 6 d^{1} F+8 \% 4 f 6 d^{3} D$ \\
\hline & 194617 & & 2 & $50 \% 4 f 6 d^{3} D+30 \% 4 f 6 d{ }^{1} D+11 \% 4 f 6 d^{3} F$ \\
\hline & 194683 & & 1 & $88 \% 4 f 6 d^{3} D+11 \% 4 f 6 d^{1} P$ \\
\hline \multirow[t]{3}{*}{194777.2} & 194757 & 20 & 5 & $78 \% 4 f 6 d^{3} H+16 \% 4 f 6 d^{1} H+6 \% 4 f 6 d^{3} G$ \\
\hline & 195303 & & 0 & $100 \% 4 \mathrm{f} 6 \mathrm{~d}^{3} \mathrm{P}$ \\
\hline & 195336 & & 1 & $80 \% 4 f 6 d^{3} P+16 \% 4 f 6 d^{1} P$ \\
\hline 196584.7 & 196539 & 45 & 4 & $58 \% 4 f 6 d^{3} G+20 \% 4 f 6 d{ }^{3} F+18 \% 4 f 6 d{ }^{1} G$ \\
\hline \multirow[t]{2}{*}{196800.0} & 196856 & -56 & 4 & $68 \% 4 f 6 d^{3} F+28 \% 4 f 6 d^{1} G$ \\
\hline & 196942 & & 3 & $32 \% 4 f 6 d^{3} D+31 \% 4 f 6 d{ }^{1} F+29 \% 4 f 6 d^{3} F$ \\
\hline 197103.7 & 197161 & -57 & 5 & $82 \% 4 f 6 d^{3} G+12 \% 4 f 6 d^{3} H+5 \% 4 f 6 d{ }^{1} H$ \\
\hline \multirow[t]{2}{*}{197196.9} & 197266 & -69 & 6 & $100 \% 4 \mathrm{f} 6 \mathrm{~d}^{3} \mathrm{H}$ \\
\hline & 197275 & & 2 & $50 \% 4 f 6 d^{3} D+34 \% 4 f 6 d^{1} D+8 \% 4 f 6 d^{3} F$ \\
\hline \multirow[t]{2}{*}{198155.4} & 197921 & 234 & 3 & $59 \% 4 \mathrm{f} 6 \mathrm{~d}^{3} \mathrm{D}+37 \% 4 \mathrm{f} 6 \mathrm{~d}^{1} \mathrm{~F}$ \\
\hline & 197989 & & 2 & $82 \% 4 f 6 d^{3} P+16 \% 4 f 6 d{ }^{1} D$ \\
\hline \multirow[t]{3}{*}{198304.7} & 198411 & -106 & 5 & $79 \% 4 f 6 d^{1} H+12 \% 4 f 6 d{ }^{3} G+9 \% 4 f 6 d{ }^{3} H$ \\
\hline & 199103 & & 1 & $72 \% 4 f 6 d{ }^{1} P+19 \% 4 f 6 d{ }^{3} P+8 \% 4 f 6 d{ }^{3} D$ \\
\hline & 199597 & & 2 & $100 \% 4 \mathrm{f} 7 \mathrm{~s}{ }^{3} \mathrm{~F}$ \\
\hline 199727.7 & 199729 & -1 & 3 & $62 \% 4 \mathrm{f} 7 \mathrm{~s}{ }^{3} \mathrm{~F}+38 \% 4 \mathrm{f} 7 \mathrm{~s}{ }^{1} \mathrm{~F}$ \\
\hline
\end{tabular}

${ }^{a}$ From Martin et al (1978).

${ }^{\mathrm{b}} \mathrm{LS}$ composition in \%. Only the first three main components larger than $5 \%$ are given.

in the semi-empirical parametric fit performed by Wyart et al (2005). This is essentially due to the fact that the latter authors considered a much more limited set of configurations $\left(4 \mathrm{f}^{2}+\right.$ $\left.4 f 6 p+5 p^{5} 4 f^{3}\right)$ in their physical model.

\section{Electric dipole transitions}

Oscillator strengths $(\log g f)$ and transition probabilities $(g A)$ computed in this work are reported in table 5 for a sample of Pr IV lines between 118 and $302 \mathrm{~nm}$. This sample is limited to transitions involving the levels below $150000 \mathrm{~cm}^{-1}$ for which $\log g f$ is greater than -2.0 .

An argument for assessing the reliability of the present results can be obtained from isoelectronic comparisons, particularly from results obtained recently in Ce III (Biémont et al 2002), the HFR + CPOL model adopted in this work being the same as that chosen for this isoelectronic ion. More precisely, radiative lifetimes of nine states in Ce III, in the energy range from 48267 to $54550 \mathrm{~cm}^{-1}$, have been measured by Li et al (2000) using the time-resolved laser-induced fluorescence (LIF) technique. The comparison 
Table 5. Computed oscillator strengths and transition probabilities in Pr IV.

\begin{tabular}{|c|c|c|c|c|c|c|c|c|c|}
\hline \multirow{2}{*}{$\begin{array}{l}\text { Wavelength }^{\mathrm{a}} \\
(\mathrm{nm})\end{array}$} & \multicolumn{3}{|c|}{ Lower level $^{\mathrm{b}}$} & \multicolumn{3}{|c|}{ Upper level $^{\mathrm{b}}$} & \multirow[b]{2}{*}{$\log g f^{\mathrm{c}}$} & \multirow{2}{*}{$\begin{array}{l}g A^{\mathrm{c}} \\
\left(\mathrm{s}^{-1}\right)\end{array}$} & \multirow{2}{*}{$\begin{array}{l}g A^{\mathrm{d}} \\
\left(\mathrm{s}^{-1}\right)\end{array}$} \\
\hline & $E\left(\mathrm{~cm}^{-1}\right)$ & Parity & $J$ & $E\left(\mathrm{~cm}^{-1}\right)$ & Parity & $J$ & & & \\
\hline 118.7761 & 61171 & (Odd) & 4 & 145363 & (Even) & 4 & -1.37 & $2.04 \mathrm{E}+08$ & \\
\hline 119.3707 & 61171 & (Odd) & 4 & 144943 & (Even) & 3 & -1.88 & $6.15 E+07$ & \\
\hline 119.3964 & 61171 & (Odd) & 4 & 144925 & (Even) & 4 & -1.05 & $4.15 E+08$ & $7.6 \mathrm{E}+08$ \\
\hline 120.1619 & 63356 & (Odd) & 3 & 146577 & (Even) & 2 & -1.90 & $5.91 E+07$ & \\
\hline 121.2802 & 64124 & (Odd) & 3 & 146577 & (Even) & 2 & -1.36 & $1.99 \mathrm{E}+08$ & \\
\hline 122.2764 & 63581 & (Odd) & 4 & 145363 & (Even) & 4 & -1.55 & $1.26 \mathrm{E}+08$ & \\
\hline 122.3983 & 63581 & (Odd) & 4 & 145281 & (Even) & 5 & -1.86 & $6.09 \mathrm{E}+07$ & \\
\hline 122.5687 & 63356 & (Odd) & 3 & 144943 & (Even) & 3 & -1.76 & $7.69 \mathrm{E}+07$ & \\
\hline 122.6397 & 61457 & (Odd) & 2 & 142997 & (Even) & 1 & -0.81 & $6.84 \mathrm{E}+08$ & \\
\hline 122.8586 & 61171 & (Odd) & 4 & 142566 & (Even) & 4 & -0.42 & $1.69 \mathrm{E}+09$ & \\
\hline 122.9067 & 63581 & (Odd) & 4 & 144943 & (Even) & 3 & -1.63 & $1.04 \mathrm{E}+08$ & \\
\hline $122.9336 *$ & 63581 & (Odd) & 4 & 144925 & (Even) & 4 & -1.59 & $1.13 \mathrm{E}+08$ & $1.4 \mathrm{E}+08$ \\
\hline 123.0691 & 65322 & (Odd) & 2 & 146577 & (Even) & 2 & -0.60 & $1.09 \mathrm{E}+09$ & \\
\hline 123.7600 & 64124 & (Odd) & 3 & 144925 & (Even) & 4 & -1.68 & $9.20 \mathrm{E}+07$ & $1.0 \mathrm{E}+08$ \\
\hline 124.8081 & 65239 & (Odd) & 5 & 145363 & (Even) & 4 & -1.63 & $1.02 \mathrm{E}+08$ & \\
\hline 124.9353 & 65239 & (Odd) & 5 & 145281 & (Even) & 5 & -1.01 & $4.15 E+08$ & \\
\hline 125.3191 & 61457 & (Odd) & 2 & 141254 & (Even) & 3 & -1.66 & $9.16 \mathrm{E}+07$ & \\
\hline $125.4347 *$ & 65640 & (Odd) & 4 & 145363 & (Even) & 4 & -1.24 & $2.45 \mathrm{E}+08$ & \\
\hline 125.5637 & 65640 & (Odd) & 4 & 145281 & (Even) & 5 & -1.07 & $3.59 E+08$ & \\
\hline 125.5943 & 65322 & (Odd) & 2 & 144943 & (Even) & 3 & -1.62 & $1.02 \mathrm{E}+08$ & \\
\hline 125.6136 & 66968 & (Odd) & 1 & 146577 & (Even) & 2 & -1.52 & $1.28 \mathrm{E}+08$ & \\
\hline $126.0981 *$ & 65640 & (Odd) & 4 & 144943 & (Even) & 3 & -1.26 & $2.31 \mathrm{E}+08$ & \\
\hline 126.2471 & 63356 & (Odd) & 3 & 142566 & (Even) & 4 & -1.38 & $1.76 \mathrm{E}+08$ & \\
\hline 126.4936 & 61171 & (Odd) & 4 & 140226 & (Even) & 4 & -1.45 & $1.49 \mathrm{E}+08$ & $3.4 \mathrm{E}+08$ \\
\hline 126.6062 & 63581 & (Odd) & 4 & 142566 & (E & 4 & -1.00 & $4.18 \mathrm{E}+08$ & \\
\hline 126.6218 & 63356 & (Odd) & 3 & 142332 & (ven) & 2 & -0.96 & $4.62 \mathrm{E}+08$ & \\
\hline 126.8319 & 66518 & (Odd) & 4 & 145363 & (Even) & 4 & -0.77 & $7.07 \mathrm{E}+08$ & \\
\hline 126.9633 & 66518 & (Odd) & 4 & 145281 & (Even) & 5 & -1.15 & $2.95 E+08$ & \\
\hline 127.0584 & 61171 & (Odd) & 4 & 139875 & (Even) & 3 & -1.18 & $2.71 E+08$ & $4.1 \mathrm{E}+08$ \\
\hline 127.5103 & 66518 & (Odd) & 4 & 144943 & (Even) & 3 & -0.10 & $3.26 \mathrm{E}+09$ & \\
\hline 127.5223 & 61457 & (Odd) & 2 & 139875 & (Even) & 3 & -1.80 & $6.44 \mathrm{E}+07$ & $4.0 \mathrm{E}+07$ \\
\hline 127.5395 & 66518 & (Odd) & 4 & 144925 & (Even) & 4 & -0.26 & $2.27 E+09$ & $3.0 \mathrm{E}+09$ \\
\hline 127.8646 & 64124 & (Odd) & 3 & 142332 & (Even) & 2 & -0.28 & $2.13 \mathrm{E}+09$ & \\
\hline 127.9337 & 68412 & (Odd) & 2 & 146577 & (Even) & 2 & -0.52 & $1.23 \mathrm{E}+09$ & \\
\hline 128.0714 & 68496 & (Odd) & 3 & 146577 & (Even) & 2 & -1.46 & $1.41 \mathrm{E}+08$ & \\
\hline $128.7405^{*}$ & 65322 & (Odd) & 2 & 142997 & (Even) & 1 & -0.87 & $5.42 \mathrm{E}+08$ & \\
\hline 128.7442 & 63581 & (Odd) & 4 & 141254 & (Even) & 3 & 0.07 & $4.70 \mathrm{E}+09$ & \\
\hline 129.0933 & 67899 & (Odd) & 5 & 145363 & (Even) & 4 & 0.08 & $4.85 \mathrm{E}+09$ & \\
\hline 129.2295 & 67899 & (Odd) & 5 & 145281 & (Even) & 5 & -0.07 & $3.40 \mathrm{E}+09$ & \\
\hline 129.3220 & 65239 & (Odd) & 5 & 142566 & (Even) & 4 & 0.10 & $5.09 \mathrm{E}+09$ & \\
\hline 129.5282 & 68078 & (Odd) & 6 & 145281 & (Even) & 5 & 0.43 & $1.07 \mathrm{E}+10$ & \\
\hline 129.6504 & 64124 & (Odd) & 3 & 141254 & (Even) & 3 & -0.57 & $1.06 \mathrm{E}+09$ & \\
\hline 129.8264 & 67899 & (Odd) & 5 & 144925 & (Even) & 4 & -1.29 & $2.05 E+08$ & $1.3 \mathrm{E}+09$ \\
\hline 129.8535 & 65322 & (Odd) & 2 & 142332 & (Even) & 2 & -0.38 & $1.65 E+09$ & \\
\hline 129.9950 & 65640 & (Odd) & 4 & 142566 & (Even) & 4 & -1.06 & $3.48 \mathrm{E}+08$ & \\
\hline 130.0899 & 63356 & (Odd) & 3 & 140226 & (Even) & 4 & -1.50 & $1.25 \mathrm{E}+08$ & $2.2 \mathrm{E}+08$ \\
\hline $130.0946 *$ & 68496 & (Odd) & 3 & 145363 & (Even) & 4 & -1.13 & $2.90 \mathrm{E}+08$ & \\
\hline 130.4712 & 63581 & (Odd) & 4 & 140226 & (Even) & 4 & -0.67 & $8.51 E+08$ & $1.1 \mathrm{E}+09$ \\
\hline 130.6860 & 63356 & (Odd) & 3 & 139875 & (Even) & 3 & -1.20 & $2.47 \mathrm{E}+08$ & $6.8 \mathrm{E}+08$ \\
\hline 130.8085 & 68496 & (Odd) & 3 & 144943 & (Even) & 3 & -0.82 & $5.85 \mathrm{E}+08$ & \\
\hline $130.9657 *$ & 63356 & (Odd) & 3 & 139712 & (Even) & 2 & -0.30 & $1.95 \mathrm{E}+09$ & \\
\hline 131.0708 & 63581 & (Odd) & 4 & 139875 & (Even) & 3 & -1.53 & $1.16 \mathrm{E}+08$ & $2.7 \mathrm{E}+08$ \\
\hline 131.4021 & 64124 & (Odd) & 3 & 140226 & (Even) & 4 & -0.97 & $4.20 \mathrm{E}+08$ & $4.1 \mathrm{E}+08$ \\
\hline 131.4961 & 66518 & (Odd) & 4 & 142566 & (Even) & 4 & -0.54 & $1.11 \mathrm{E}+09$ & \\
\hline 131.5279 & 66968 & (Odd) & 1 & 142997 & (Even) & 1 & -0.57 & $1.04 \mathrm{E}+09$ & \\
\hline 131.6961 & 65322 & (Odd) & 2 & 141254 & (Even) & 3 & -0.90 & $4.78 \mathrm{E}+08$ & \\
\hline 131.8879 & 70755 & (Odd) & 1 & 146577 & (Even) & 2 & -1.47 & $1.30 \mathrm{E}+08$ & \\
\hline 132.0101 & 64124 & (Odd) & 3 & 139875 & (Even) & 3 & -0.26 & $2.09 \mathrm{E}+09$ & $1.8 \mathrm{E}+09$ \\
\hline 132.0699 & 61457 & (Odd) & 2 & 137175 & (Even) & 2 & -0.15 & $2.68 \mathrm{E}+09$ & \\
\hline 132.1355 & 61171 & (Odd) & 4 & 136851 & (Even) & 3 & 0.10 & $4.76 \mathrm{E}+09$ & \\
\hline 132.2506 & 65640 & (Odd) & 4 & 141254 & (Even) & 3 & -1.65 & $8.65 E+07$ & \\
\hline 132.6376 & 61457 & (Odd) & 2 & 136851 & (Even) & 3 & -0.74 & $6.88 \mathrm{E}+08$ & \\
\hline 133.3573 & 65239 & (Odd) & 5 & 140226 & (Even) & 4 & -0.06 & $3.29 E+09$ & $3.8 \mathrm{E}+09$ \\
\hline
\end{tabular}


Table 5. (Continued.)

\begin{tabular}{|c|c|c|c|c|c|c|c|c|c|}
\hline \multirow{2}{*}{$\begin{array}{l}\text { Wavelength } \\
(\mathrm{nm})\end{array}$} & \multicolumn{3}{|c|}{ Lower level $^{\mathrm{b}}$} & \multicolumn{3}{|c|}{ Upper level $^{\mathrm{b}}$} & \multirow[b]{2}{*}{$\log g f^{\mathrm{c}}$} & \multirow{2}{*}{$\begin{array}{l}g A^{\mathrm{c}} \\
\left(\mathrm{s}^{-1}\right)\end{array}$} & \multirow{2}{*}{$\begin{array}{l}g A^{\mathrm{d}} \\
\left(\mathrm{s}^{-1}\right)\end{array}$} \\
\hline & $E\left(\mathrm{~cm}^{-1}\right)$ & Parity & $J$ & $E\left(\mathrm{~cm}^{-1}\right)$ & Parity & $J$ & & & \\
\hline 133.5963 & 71725 & (Odd) & 3 & 146577 & (Even) & 2 & -0.45 & $1.32 \mathrm{E}+09$ & \\
\hline 133.9288 & 67899 & (Odd) & 5 & 142566 & (Even) & 4 & -0.41 & $1.44 \mathrm{E}+09$ & \\
\hline 134.0738 & 65640 & (Odd) & 4 & 140226 & (Even) & 4 & -0.14 & $2.69 \mathrm{E}+09$ & $2.9 \mathrm{E}+09$ \\
\hline 134.0738 & 68412 & (Odd) & 2 & 142997 & (Even) & 1 & -1.22 & $2.24 \mathrm{E}+08$ & \\
\hline 134.1315 & 65322 & (Odd) & 2 & 139875 & (Even) & 3 & -1.53 & $1.08 \mathrm{E}+08$ & $2.5 \mathrm{E}+08$ \\
\hline 134.4229 & 72185 & (Odd) & 2 & 146577 & (Even) & 2 & -0.77 & $6.27 \mathrm{E}+08$ & \\
\hline $134.4264 *$ & 65322 & (Odd) & 2 & 139712 & (Even) & 2 & -1.55 & $1.03 \mathrm{E}+08$ & \\
\hline 134.7066 & 65640 & (Odd) & 4 & 139875 & (Even) & 3 & -0.41 & $1.43 \mathrm{E}+09$ & $2.2 \mathrm{E}+09$ \\
\hline $135.0068 *$ & 68496 & (Odd) & 3 & 142566 & (Even) & 4 & -1.06 & $3.15 E+08$ & \\
\hline 135.2811 & 68412 & (Odd) & 2 & 142332 & (Even) & 2 & -0.78 & $6.01 E+08$ & \\
\hline 135.4350 & 68496 & (Odd) & 3 & 142332 & (Even) & 2 & -0.81 & $5.64 \mathrm{E}+08$ & \\
\hline 135.4663 & 63356 & (Odd) & 3 & 137175 & (Even) & 2 & -0.47 & $1.24 \mathrm{E}+09$ & \\
\hline 135.6705 & 66518 & (Odd) & 4 & 140226 & (Even) & 4 & -1.18 & $2.38 \mathrm{E}+08$ & $1.2 \mathrm{E}+08$ \\
\hline 135.7989 & 71725 & (Odd) & 3 & 145363 & (Even) & 4 & -0.94 & $4.17 E+08$ & \\
\hline 136.0639 & 63356 & (Odd) & 3 & 136851 & (Even) & 3 & -0.22 & $2.19 \mathrm{E}+09$ & \\
\hline 136.3190 & 66518 & (Odd) & 4 & 139875 & (Even) & 3 & -1.50 & $1.14 \mathrm{E}+08$ & $3.0 \mathrm{E}+08$ \\
\hline 136.4811 & 63581 & (Odd) & 4 & 136851 & (Even) & 3 & -0.85 & $5.10 \mathrm{E}+08$ & \\
\hline 136.5773 & 71725 & (Odd) & 3 & 144943 & (Even) & 3 & -0.13 & $2.65 E+09$ & \\
\hline 136.6107 & 71725 & (Odd) & 3 & 144925 & (Even) & 4 & -1.07 & $3.04 \mathrm{E}+08$ & $8.0 \mathrm{E}+08$ \\
\hline 136.8895 & 64124 & (Odd) & 3 & 137175 & (Even) & 2 & -1.40 & $1.41 E+08$ & \\
\hline 137.4414 & 68496 & (Odd) & 3 & 141254 & (Even) & 3 & -0.43 & $1.31 E+09$ & \\
\hline 137.4414 & 72185 & (Odd) & 2 & 144943 & (Even) & 3 & -0.22 & $2.13 \mathrm{E}+09$ & \\
\hline $137.4682 *$ & 66968 & (Odd) & 1 & 139712 & (Even) & 2 & -1.26 & $1.94 \mathrm{E}+08$ & \\
\hline 138.2617 & 67899 & (Odd) & 5 & 140226 & (Even) & 4 & -0.44 & $1.28 \mathrm{E}+09$ & $1.7 \mathrm{E}+09$ \\
\hline 138.4234 & 70755 & (Odd) & 1 & 142997 & (Even) & 1 & -0.67 & $7.46 \mathrm{E}+08$ & \\
\hline 138.5914 & 70843 & (Odd) & 0 & 142997 & (Even) & 1 & -0.68 & $7.30 \mathrm{E}+08$ & \\
\hline 139.4106 & 68496 & (Odd) & 3 & 140226 & (Even) & 4 & -0.68 & $7.21 \mathrm{E}+08$ & $8.1 \mathrm{E}+08$ \\
\hline 139.7110 & 70755 & (Odd) & 1 & 142332 & (Even) & 2 & -0.52 & $1.05 E+09$ & \\
\hline 139.8029 & 65322 & (Odd) & 2 & 136851 & (Even) & 3 & -1.63 & $7.88 \mathrm{E}+07$ & \\
\hline 139.9313 & 68412 & (Odd) & 2 & 139875 & (Even) & 3 & -0.36 & $1.50 \mathrm{E}+09$ & $1.8 \mathrm{E}+09$ \\
\hline 140.0961 & 68496 & (Odd) & 3 & 139875 & (Even) & 3 & -0.54 & $9.77 \mathrm{E}+08$ & $1.2 \mathrm{E}+09$ \\
\hline 141.2182 & 72185 & (Odd) & 2 & 142997 & (Even) & 1 & -1.97 & $3.58 \mathrm{E}+07$ & \\
\hline 142.4357 & 66968 & (Odd) & 1 & 137175 & (Even) & 2 & -0.57 & $8.76 \mathrm{E}+08$ & \\
\hline 142.5587 & 72185 & (Odd) & 2 & 142332 & (Even) & 2 & -1.23 & $1.92 \mathrm{E}+08$ & \\
\hline 142.6594 & 75266 & (Odd) & 5 & 145363 & (Even) & 4 & -0.39 & $1.35 E+09$ & \\
\hline 142.8253 & 75266 & (Odd) & 5 & 145281 & (Even) & 5 & -1.89 & $4.22 \mathrm{E}+07$ & \\
\hline 143.5555 & 75266 & (Odd) & 5 & 144925 & (Even) & 4 & 0.03 & $3.45 E+09$ & $5.6 \mathrm{E}+09$ \\
\hline 143.8244 & 71725 & (Odd) & 3 & 141254 & (Even) & 3 & -1.24 & $1.87 \mathrm{E}+08$ & \\
\hline 144.7829 & 72185 & (Odd) & 2 & 141254 & (Even) & 3 & -1.33 & $1.49 \mathrm{E}+08$ & \\
\hline $145.0190 *$ & 70755 & (Odd) & 1 & 139712 & (Even) & 2 & -1.57 & $8.53 \mathrm{E}+07$ & \\
\hline 146.2942 & 68496 & (Odd) & 3 & 136851 & (Even) & 3 & -1.18 & $2.04 \mathrm{E}+08$ & \\
\hline 146.7342 & 71725 & (Odd) & 3 & 139875 & (Even) & 3 & -1.52 & $9.28 \mathrm{E}+07$ & $1.2 \mathrm{E}+08$ \\
\hline 147.4913 & 78776 & (Odd) & 1 & 146577 & (Even) & 2 & -0.25 & $1.75 E+09$ & \\
\hline 147.7321 & 72185 & (Odd) & 2 & 139875 & (Even) & 3 & -0.92 & $3.66 \mathrm{E}+08$ & $4.1 \mathrm{E}+08$ \\
\hline 148.5880 & 75266 & (Odd) & 5 & 142566 & (Even) & 4 & -0.64 & $6.83 E+08$ & \\
\hline 150.5578 & 70755 & (Odd) & 1 & 137175 & (Even) & 2 & -1.33 & $1.38 \mathrm{E}+08$ & \\
\hline 152.0977 & 2152 & (Even) & 5 & 67899 & (Odd) & 5 & -1.64 & $6.61 E+07$ & \\
\hline 152.3462 & 0 & (Even) & 4 & 65640 & (Odd) & 4 & -1.72 & $5.44 E+07$ & \\
\hline 153.0352 & 9921 & (Even) & 4 & 75266 & (Odd) & 5 & -1.99 & $2.96 \mathrm{E}+07$ & \\
\hline $153.5483 *$ & 71725 & (Odd) & 3 & 136851 & (Even) & 3 & -1.47 & $9.70 \mathrm{E}+07$ & \\
\hline 153.9404 & 75266 & (Odd) & 5 & 140226 & (Even) & 4 & -0.62 & $6.73 E+08$ & $8.1 \mathrm{E}+08$ \\
\hline 155.3617 & 2152 & (Even) & 5 & 66518 & (Odd) & 4 & -1.51 & $8.60 \mathrm{E}+07$ & \\
\hline 155.9489 & 0 & (Even) & 4 & 64124 & (Odd) & 3 & -1.50 & $8.64 \mathrm{E}+07$ & \\
\hline 157.0133 & 4389 & (Even) & 6 & 68078 & (Odd) & 6 & -0.79 & $4.36 \mathrm{E}+08$ & \\
\hline 157.2804 & 0 & (Even) & 4 & 63581 & (Oodd) & 4 & -1.49 & $8.63 \mathrm{E}+07$ & \\
\hline $157.3435^{*}$ & 78776 & (Odd) & 1 & 142332 & (Even) & 2 & -1.56 & $7.45 E+07$ & \\
\hline 157.4547 & 4389 & (Even) & 6 & 67899 & (Odd) & 5 & -0.18 & $1.77 \mathrm{E}+09$ & \\
\hline 157.5101 & 2152 & (Even) & 5 & 65640 & (Odd) & 4 & -0.31 & $1.32 \mathrm{E}+09$ & \\
\hline 157.6911 & 4997 & (Even) & 2 & 68412 & (Odd) & 2 & -1.65 & $5.98 \mathrm{E}+07$ & \\
\hline 157.8381 & 0 & (Even) & 4 & 63356 & (Odd) & 3 & -0.40 & $1.06 \mathrm{E}+09$ & \\
\hline 158.5100 & 2152 & (Even) & 5 & 65239 & (Odd) & 5 & -0.88 & $3.45 E+08$ & \\
\hline 161.0816 & 6415 & (Even) & 3 & 68496 & (Odd) & 3 & -1.68 & $5.40 \mathrm{E}+07$ & \\
\hline 161.3002 & 6415 & (Even) & 3 & 68412 & (Odd) & 2 & -0.86 & $3.59 \mathrm{E}+08$ & \\
\hline
\end{tabular}


Table 5. (Continued.)

\begin{tabular}{|c|c|c|c|c|c|c|c|c|c|}
\hline \multirow{2}{*}{$\begin{array}{l}\text { Wavelength }^{\mathrm{a}} \\
(\mathrm{nm})\end{array}$} & \multicolumn{3}{|c|}{ Lower level $^{\mathrm{b}}$} & \multicolumn{3}{|c|}{ Upper level $^{\mathrm{b}}$} & \multirow[b]{2}{*}{$\log g f^{\mathrm{c}}$} & \multirow{2}{*}{$\begin{array}{l}g A^{\mathrm{c}} \\
\left(\mathrm{s}^{-1}\right)\end{array}$} & \multirow{2}{*}{$\begin{array}{l}g A^{\mathrm{d}} \\
\left(\mathrm{s}^{-1}\right)\end{array}$} \\
\hline & $E\left(\mathrm{~cm}^{-1}\right)$ & Parity & $J$ & $E\left(\mathrm{~cm}^{-1}\right)$ & Parity & $J$ & & & \\
\hline 161.3654 & 4997 & (Even) & 2 & 66968 & (Odd) & 1 & -0.95 & $2.87 \mathrm{E}+08$ & \\
\hline 161.8032 & 9921 & (Even) & 4 & 71725 & (Odd) & 3 & -0.49 & $8.23 \mathrm{E}+08$ & \\
\hline 162.2305 & 6855 & (Even) & 4 & 68496 & (Odd) & 3 & -0.57 & $6.84 \mathrm{E}+08$ & \\
\hline 162.7552 & 17334 & (Even) & 2 & 78776 & (Odd) & 1 & -1.20 & $1.60 \mathrm{E}+08$ & \\
\hline 162.7911 & 2152 & (Even) & 5 & 63581 & (Odd) & 4 & -1.70 & $4.99 \mathrm{E}+07$ & \\
\hline 163.4770 & 0 & (Even) & 4 & 61171 & (Odd) & 4 & -1.10 & $1.99 \mathrm{E}+08$ & \\
\hline 163.8151 & 6855 & (Even) & 4 & 67899 & (Odd) & 5 & -1.61 & $6.11 E+07$ & \\
\hline $164.1082 *$ & 78776 & (Odd) & 1 & 139712 & (Even) & 2 & -1.98 & $2.60 \mathrm{E}+07$ & \\
\hline 167.6084 & 6855 & (Even) & 4 & 66518 & (Odd) & 4 & -1.20 & $1.51 \mathrm{E}+08$ & \\
\hline 168.8489 & 6415 & (Even) & 3 & 65640 & (Odd) & 4 & -1.45 & $8.27 \mathrm{E}+07$ & \\
\hline 169.7611 & 6415 & (Even) & 3 & 65322 & (Odd) & 2 & -1.39 & $9.46 \mathrm{E}+07$ & \\
\hline 170.1112 & 6855 & (Even) & 4 & 65640 & (Odd) & 4 & -1.90 & $2.88 \mathrm{E}+07$ & \\
\hline $171.2368 *$ & 78776 & (Odd) & 1 & 137175 & (Even) & 2 & -1.60 & $5.76 \mathrm{E}+07$ & \\
\hline 171.3526 & 4997 & (Even) & 2 & 63356 & (Odd) & 3 & -1.56 & $6.17 \mathrm{E}+07$ & \\
\hline 173.2859 & 6415 & (Even) & 3 & 64124 & (Odd) & 3 & -0.79 & $3.57 \mathrm{E}+08$ & \\
\hline 174.6158 & 6855 & (Even) & 4 & 64124 & (Odd) & 3 & -1.75 & $3.88 \mathrm{E}+07$ & \\
\hline 176.2861 & 6855 & (Even) & 4 & 63581 & (Odd) & 4 & -1.06 & $1.85 \mathrm{E}+08$ & \\
\hline 176.6883 & 9921 & (Even) & 4 & 66518 & (Odd) & 4 & -0.80 & $3.40 \mathrm{E}+08$ & \\
\hline 177.1137 & 4997 & (Even) & 2 & 61457 & (Odd) & 2 & -0.93 & $2.53 \mathrm{E}+08$ & \\
\hline 179.8058 & 23161 & (Even) & 2 & 78776 & (Odd) & 1 & -1.82 & $3.14 \mathrm{E}+07$ & \\
\hline 183.8573 & 17334 & (Even) & 2 & 71725 & (Odd) & 3 & -1.87 & $2.65 \mathrm{E}+07$ & \\
\hline 184.1082 & 6855 & (Even) & 4 & 61171 & (Odd) & 4 & -1.27 & $1.05 \mathrm{E}+08$ & \\
\hline 186.3615 & 9921 & (Even) & 4 & 63581 & (Odd) & 4 & -1.82 & $2.90 \mathrm{E}+07$ & \\
\hline 187.1931 & 17334 & (Even) & 2 & 70755 & (Odd) & 1 & -1.81 & $2.97 \mathrm{E}+07$ & \\
\hline 188.4870 & 22212 & (Even) & 6 & 75266 & (Odd) & 5 & -0.12 & $1.43 \mathrm{E}+09$ & \\
\hline 195.1234 & 9921 & (Even) & 4 & 61171 & (Odd) & 4 & -1.66 & $3.90 \mathrm{E}+07$ & \\
\hline 195.4609 & 17334 & (Even) & 2 & 68496 & (Odd) & 3 & -1.56 & $4.81 \mathrm{E}+07$ & \\
\hline 195.7825 & 17334 & (Even) & 2 & 68412 & (Odd) & 2 & -1.79 & $2.82 \mathrm{E}+07$ & \\
\hline 199.2924 & 22007 & (Even) & 1 & 72185 & (Odd) & 2 & -1.45 & $5.95 \mathrm{E}+07$ & \\
\hline 202.5062 & 21390 & (Even) & 0 & 70755 & (Odd) & 1 & -1.57 & $4.40 \mathrm{E}+07$ & \\
\hline 203.9150 & 23161 & (Even) & 2 & 72185 & (Odd) & 2 & -0.87 & $2.17 \mathrm{E}+08$ & \\
\hline 204.7047 & 22007 & (Even) & 1 & 70843 & (Odd) & 0 & -1.47 & $5.40 \mathrm{E}+07$ & \\
\hline 205.0726 & 22007 & (Even) & 1 & 70755 & (Odd) & 1 & -1.54 & $4.53 \mathrm{E}+07$ & \\
\hline 205.8485 & 23161 & (Even) & 2 & 71725 & (Odd) & 3 & -1.49 & $5.10 \mathrm{E}+07$ & \\
\hline 208.3228 & 17334 & (Even) & 2 & 65322 & (Odd) & 2 & -0.97 & $1.66 \mathrm{E}+08$ & \\
\hline 210.0418 & 23161 & (Even) & 2 & 70755 & (Odd) & 1 & -1.52 & $4.55 \mathrm{E}+07$ & \\
\hline 215.4313 & 22007 & (Even) & 1 & 68412 & (Odd) & 2 & -1.39 & $5.87 \mathrm{E}+07$ & \\
\hline 219.3370 & 21390 & (Even) & 0 & 66968 & (Odd) & 1 & -1.71 & $2.67 \mathrm{E}+07$ & \\
\hline 220.5131 & 23161 & (Even) & 2 & 68496 & (Odd) & 3 & -1.55 & $3.85 \mathrm{E}+07$ & \\
\hline $225.1584 *$ & 100544 & (Odd) & 3 & 144943 & (Even) & 3 & -1.06 & $1.14 \mathrm{E}+08$ & \\
\hline 225.250 & 100544 & (Odd) & 3 & 144925 & (Even) & 4 & -0.52 & $3.91 \mathrm{E}+08$ & $2.7 \mathrm{E}+08$ \\
\hline 226.5696 & 17334 & (Even) & 2 & 61457 & (Odd) & 2 & -1.75 & $2.34 \mathrm{E}+07$ & \\
\hline 233.446 & 103754 & (Odd) & 3 & 146577 & (Even) & 2 & 0.26 & $2.23 \mathrm{E}+09$ & \\
\hline 233.908 & 100258 & (Odd) & 2 & 142997 & (Even) & 1 & 0.04 & $1.34 \mathrm{E}+09$ & \\
\hline 237.505 & 103271 & (Odd) & 4 & 145363 & (Even) & 4 & -0.10 & $9.55 \mathrm{E}+08$ & \\
\hline 237.609 & 100258 & (Odd) & 2 & 142332 & (Even) & 2 & 0.03 & $1.28 \mathrm{E}+09$ & \\
\hline 237.898 & 100544 & (Odd) & 3 & 142566 & (Even) & 4 & 0.47 & $3.47 \mathrm{E}+09$ & \\
\hline 237.966 & 103271 & (Odd) & 4 & 145281 & (Even) & 5 & 0.61 & $4.81 \mathrm{E}+09$ & \\
\hline 239.232 & 100544 & (Odd) & 3 & 142332 & (Even) & 2 & -0.15 & $8.30 \mathrm{E}+08$ & \\
\hline 239.897 & 103271 & (Odd) & 4 & 144943 & (Even) & 3 & 0.02 & $1.23 \mathrm{E}+09$ & \\
\hline 240.000 & 103271 & (Odd) & 4 & 144925 & (Even) & 4 & -0.49 & $3.72 \mathrm{E}+08$ & $1.6 \mathrm{E}+09$ \\
\hline 240.259 & 103754 & (Odd) & 3 & 145363 & (Even) & 4 & -0.12 & $8.76 \mathrm{E}+08$ & \\
\hline 242.707 & 103754 & (Odd) & 3 & 144943 & (Even) & 3 & 0.15 & $1.60 \mathrm{E}+09$ & \\
\hline 242.813 & 103754 & (Odd) & 3 & 144925 & (Even) & 4 & 0.13 & $1.51 \mathrm{E}+09$ & $3.1 E+09$ \\
\hline 243.857 & 100258 & (Odd) & 2 & 141254 & (Even) & 3 & 0.08 & $1.35 \mathrm{E}+09$ & \\
\hline 245.564 & 100544 & (Odd) & 3 & 141254 & (Even) & 3 & -0.12 & $8.29 \mathrm{E}+08$ & \\
\hline $251.9272 *$ & 100544 & (Odd) & 3 & 140226 & (Even) & 4 & -1.01 & $1.03 \mathrm{E}+08$ & $1.0 \mathrm{E}+08$ \\
\hline 252.344 & 100258 & (Odd) & 2 & 139875 & (Even) & 3 & -1.01 & $1.01 \mathrm{E}+08$ & $9.0 \mathrm{E}+07$ \\
\hline $254.1731 *$ & 100544 & (Odd) & 3 & 139875 & (Even) & 3 & -0.86 & $1.43 \mathrm{E}+08$ & $1.8 \mathrm{E}+08$ \\
\hline 254.411 & 103271 & (Odd) & 4 & 142566 & (Even) & 4 & -0.66 & $2.25 \mathrm{E}+08$ & \\
\hline $255.2342 *$ & 100544 & (Odd) & 3 & 139712 & (Even) & 2 & -0.75 & $1.82 \mathrm{E}+08$ & \\
\hline $257.5739 *$ & 103754 & (Odd) & 3 & 142566 & (Even) & 4 & -1.42 & $3.77 \mathrm{E}+07$ & \\
\hline 263.197 & 103271 & (Odd) & 4 & 141254 & (Even) & 3 & -0.96 & $1.06 \mathrm{E}+08$ & \\
\hline
\end{tabular}


Table 5. (Continued.)

\begin{tabular}{|c|c|c|c|c|c|c|c|c|c|}
\hline \multirow{2}{*}{$\begin{array}{l}\text { Wavelength }^{\mathrm{a}} \\
(\mathrm{nm})\end{array}$} & \multicolumn{3}{|c|}{ Lower level $^{\mathrm{b}}$} & \multicolumn{3}{|c|}{ Upper level $^{\mathrm{b}}$} & \multirow[b]{2}{*}{$\log g f^{\mathrm{c}}$} & \multirow{2}{*}{$\begin{array}{l}g A^{\mathrm{c}} \\
\left(\mathrm{s}^{-1}\right)\end{array}$} & \multirow{2}{*}{$\begin{array}{l}g A^{\mathrm{d}} \\
\left(\mathrm{s}^{-1}\right)\end{array}$} \\
\hline & $E\left(\mathrm{~cm}^{-1}\right)$ & Parity & $J$ & $E\left(\mathrm{~cm}^{-1}\right)$ & Parity & $J$ & & & \\
\hline 266.585 & 103754 & (Odd) & 3 & 141254 & (Eve & 3 & -0.60 & $2.35 E+08$ & \\
\hline 270.519 & 103271 & (Odd) & 4 & 140226 & (Even) & 4 & 0.27 & $1.68 \mathrm{E}+09$ & $2.2 \mathrm{E}+09$ \\
\hline 270.801 & 100258 & (Odd) & 2 & 137175 & (Even) & 2 & -0.14 & $6.61 \mathrm{E}+08$ & \\
\hline 272.911 & 100544 & (Odd) & 3 & 137175 & (Even) & 2 & -0.11 & $7.01 E+08$ & \\
\hline 273.112 & 103271 & (Odd) & 4 & 139875 & (Even) & 3 & 0.03 & $9.63 \mathrm{E}+08$ & $1.3 \mathrm{E}+09$ \\
\hline 273.201 & 100258 & (Odd) & 2 & 136851 & (Even) & 3 & -0.01 & $8.68 \mathrm{E}+08$ & \\
\hline 274.100 & 103754 & (Odd) & 3 & 140226 & (Even) & 4 & 0.00 & $8.81 E+08$ & $1.2 \mathrm{E}+09$ \\
\hline 275.347 & 100544 & (Odd) & 3 & 136851 & (Even) & 3 & 0.11 & $1.15 E+09$ & \\
\hline 276.760 & 103754 & (Odd) & 3 & 139875 & (Even) & 3 & -0.07 & $7.32 \mathrm{E}+08$ & $1.1 \mathrm{E}+09$ \\
\hline 302.052 & 103754 & (Odd) & 3 & 136851 & (Even) & 3 & -1.37 & $3.15 E+07$ & \\
\hline
\end{tabular}

a Air (above $200 \mathrm{~nm}$ ) and vacuum (below $200 \mathrm{~nm}$ ) wavelengths observed by Sugar (1965). Starred values were deduced from experimental energy levels compiled by Martin et al (1978).

${ }^{\mathrm{b}}$ From Martin et al (1978).

c This work.

${ }^{\mathrm{d}}$ From Wyart et al (2005).

Table 6. Transition probabilities for forbidden lines within the $4 \mathrm{f}^{2}$ ground-state configuration of $\operatorname{Pr}$ IV.

\begin{tabular}{|c|c|c|c|c|}
\hline Air wavelength $^{\mathrm{a}}(\mathrm{nm})$ & Transition & Type $^{\text {b }}$ & $g A$ (This work) $\left(\mathrm{s}^{-1}\right)$ & $g A(\text { Other })^{\mathrm{c}}\left(\mathrm{s}^{-1}\right)$ \\
\hline 450.0902 & ${ }^{3} \mathrm{H}_{4}-{ }^{1} \mathrm{I}_{6}$ & E2 & $1.63 \mathrm{E}-02$ & $2.11 \mathrm{E}-02$ \\
\hline 498.3791 & ${ }^{3} \mathrm{H}_{5}-{ }^{1} \mathrm{I}_{6}$ & M1 & $6.42 \mathrm{E}+00$ & $6.29 \mathrm{E}+00$ \\
\hline 550.3866 & ${ }^{3} F_{2}-{ }^{3} P_{2}$ & $\mathrm{M} 1+\mathrm{E} 2$ & $6.84 \mathrm{E}-02$ & 6.49E-02 \\
\hline 560.9344 & ${ }^{3} \mathrm{H}_{6}-{ }^{1} \mathrm{I}_{6}$ & M1 & $6.31 \mathrm{E}+00$ & $6.14 \mathrm{E}+00$ \\
\hline 587.6972 & ${ }^{3} \mathrm{~F}_{2}-{ }^{3} \mathrm{P}_{1}$ & $\mathrm{M} 1+\mathrm{E} 2$ & $1.82 \mathrm{E}-01$ & $9.11 \mathrm{E}-02$ \\
\hline 597.0146 & ${ }^{3} \mathrm{~F}_{2}-{ }^{3} \mathrm{P}_{2}$ & $\mathrm{M} 1+\mathrm{E} 2$ & 8.31E-01 & $8.00 \mathrm{E}-01$ \\
\hline 609.8402 & ${ }^{3} \mathrm{~F}_{2}-{ }^{3} \mathrm{P}_{0}$ & E2 & $1.10 \mathrm{E}-01$ & $2.78 \mathrm{E}-03$ \\
\hline 613.1068 & ${ }^{3} \mathrm{~F}_{4}-{ }^{3} \mathrm{P}_{2}$ & $\mathrm{E} 2$ & $1.61 \mathrm{E}-01$ & $3.06 \mathrm{E}-02$ \\
\hline 641.1683 & ${ }^{3} \mathrm{~F}_{3}-{ }^{3} \mathrm{P}_{1}$ & $\mathrm{E} 2$ & $1.67 \mathrm{E}-01$ & $1.94 \mathrm{E}-02$ \\
\hline 650.9979 & ${ }^{3} \mathrm{~F}_{4}-{ }^{1} \mathrm{I}_{6}$ & $\mathrm{E} 2$ & $2.50 \mathrm{E}-02$ & $2.87 \mathrm{E}-02$ \\
\hline 755.1150 & ${ }^{1} \mathrm{G}_{4}-{ }^{3} \mathrm{P}_{2}$ & $\mathrm{E} 2$ & $8.68 \mathrm{E}-02$ & $1.67 \mathrm{E}-02$ \\
\hline 810.2958 & ${ }^{3} \mathrm{~F}_{2}-{ }^{1} \mathrm{D}_{2}$ & M1 & $3.82 \mathrm{E}+00$ & $3.69 \mathrm{E}+00$ \\
\hline 813.4261 & ${ }^{1} \mathrm{G}_{4}-{ }^{1} \mathrm{I}_{6}$ & E2 & $2.28 \mathrm{E}-02$ & $2.57 \mathrm{E}-02$ \\
\hline 915.5709 & ${ }^{3} \mathrm{~F}_{3}-{ }^{1} \mathrm{D}_{2}$ & M1 & $5.01 \mathrm{E}+00$ & $4.77 \mathrm{E}+00$ \\
\hline 953.9696 & ${ }^{3} \mathrm{~F}_{4}-{ }^{1} \mathrm{D}_{2}$ & $\mathrm{E} 2$ & $2.31 \mathrm{E}-02$ & 4.73E-03 \\
\hline 1007.6623 & ${ }^{3} \mathrm{H}_{4}-{ }^{1} \mathrm{G}_{4}$ & M1 & $1.56 \mathrm{E}+00$ & $1.51 \mathrm{E}+00$ \\
\hline 1286.7901 & ${ }^{3} \mathrm{H}_{5}-{ }^{1} \mathrm{G}_{4}$ & M1 & $1.80 \mathrm{E}+00$ & $1.76 \mathrm{E}+00$ \\
\hline 1458.4438 & ${ }^{3} \mathrm{H}_{4}-{ }^{3} \mathrm{~F}_{4}$ & M1 & $1.39 \mathrm{E}+00$ & $1.37 \mathrm{E}+00$ \\
\hline 1558.3623 & ${ }^{3} \mathrm{H}_{4}-{ }^{3} \mathrm{~F}_{3}$ & M1 & 4.27E-02 & 4.39E-02 \\
\hline 1715.9100 & ${ }^{1} \mathrm{D}_{2}-{ }^{3} \mathrm{P}_{2}$ & M1 & $3.09 \mathrm{E}+00$ & \\
\hline 2125.8758 & ${ }^{3} \mathrm{H}_{5}-{ }^{3} \mathrm{~F}_{4}$ & M1 & 4.49E-01 & \\
\hline 2139.3369 & ${ }^{1} \mathrm{D}_{2}-{ }^{3} \mathrm{P}_{1}$ & M1 & $5.43 \mathrm{E}-01$ & \\
\hline 2851.4754 & ${ }^{3} \mathrm{~F}_{3}-{ }^{1} \mathrm{G}_{4}$ & M1 & $2.70 \mathrm{E}+00$ & \\
\hline 3260.1682 & ${ }^{3} \mathrm{~F}_{4}-{ }^{1} \mathrm{G}_{4}$ & M1 & $1.97 \mathrm{E}+00$ & \\
\hline 4469.0540 & ${ }^{3} \mathrm{H}_{5}-{ }^{3} \mathrm{H}_{6}$ & M1 & $3.22 \mathrm{E}+00$ & \\
\hline 4645.3791 & ${ }^{3} \mathrm{H}_{4}-{ }^{3} \mathrm{H}_{5}$ & M1 & $2.72 \mathrm{E}+00$ & $2.60 \mathrm{E}+00$ \\
\hline 7047.1330 & ${ }^{3} F_{2}-{ }^{3} F_{3}$ & M1 & $2.72 \mathrm{E}+00$ & \\
\hline 8669.5351 & ${ }^{3} \mathrm{P}_{1}-{ }^{3} \mathrm{P}_{2}$ & M1 & $1.16 \mathrm{E}-01$ & \\
\hline 16185.9865 & ${ }^{3} \mathrm{P}_{0}-{ }^{3} \mathrm{P}_{1}$ & M1 & $1.40 \mathrm{E}-02$ & \\
\hline 22746.4098 & ${ }^{3} F_{3}-{ }^{3} F_{4}$ & M1 & $1.36 \mathrm{E}-02$ & \\
\hline
\end{tabular}

of these accurate laboratory measurements with the HFR + CPOL calculations of Biémont et al (2002) has shown that the computed values were in excellent agreement (in all cases within the experimental error bars, i.e. 5\%) with the experimental results. Consequently, a similar accuracy can also be expected for the radiative parameters obtained in $\mathrm{Pr}$ IV, at least for the most intense transitions.

On the theoretical side, some data for electric dipole radiation in Pr IV were published by Sen and Puri (1989) and Stanek and Migdalek (2004) who limited their investigations 
to the $6 s^{2}{ }^{1} \mathrm{~S}_{0}-6 \mathrm{~s} 6 \mathrm{p}{ }^{1,3} \mathrm{P}_{1}$ transitions (not yet observed experimentally). When comparing our results for ${ }^{1} \mathrm{~S}_{0}-{ }^{1} \mathrm{P}_{1}$ (log $g f=0.30)$ and ${ }^{1} \mathrm{~S}_{0}-{ }^{3} \mathrm{P}_{1}(\log g f=-2.21)$, we found a rather good agreement with those obtained by Stanek and Migdalek (2004) using a relativistic multiconfiguration Dirac-Fock with the CPOL model $(\log g f=0.36$ and -2.38 , respectively), a larger discrepancy being observed with the local spin density result of Sen and Puri (1989) for the ${ }^{1} \mathrm{~S}_{0}-{ }^{1} \mathrm{P}_{1}$ transition $(\log g f=0.42)$. However, it is worth noting that the results presented by those authors were more of qualitative than quantitative character due to the very limited treatment of intravalence and core-valence correlations. Transition probabilities for some $4 \mathrm{f} 5 \mathrm{~d}-4 \mathrm{f} 6 \mathrm{p}$ and 4f6s-4f6p lines in the Pr IV spectrum were also computed by Wyart et al (2005) using a semi-empirical HFR approach. Their values are reported in table 5 for comparison. We can see that our results agree with those from Wyart et al within a factor of 2 on average. The main reason for this discrepancy is of course related to the different physical models used, the one considered in our work (including a large set of interacting configurations plus CPOL effects) is much more extended than the calculation of Wyart et al carried out with a very limited number of configurations.

\section{Forbidden transitions}

Part of the unusual spectroscopic properties of triply ionized lanthanides results from the shielding of the $4 \mathrm{f}$ orbitals by the filled $5 s^{2}$ and $5 p^{6}$ sub-shells. Each of these elements has very characteristic emission lines in the visible and near infrared range due to $4 \mathrm{f} \rightarrow 4 \mathrm{f}$ transitions. These transitions, forbidden by the electric dipole selection rules, result in very long lived excited states, with typical luminescence lifetimes on the micro- to millisecond timescale. These long lifetimes facilitate 'time-gated' emission experiments which lead to drastic improvement of signal-to-noise ratios compared with more traditional steady-state measurements by removing short lived emission and scattered excitation. For this reason, transition probabilities for magnetic dipole (M1) and electric quadrupole (E2) lines within the $4 \mathrm{f}^{2}$ configuration of Pr IV were also calculated in this work.

Our results are presented in table 6 for lines with $g A$ values greater than $0.01 \mathrm{~s}^{-1}$. They are also compared, in the same table, with the data recently published by Dodson and Zia (2012). These latter authors obtained emission rates for some M1 and E2 transitions in all triply ionized lanthanides using a detailed free ion Hamiltonian, including electrostatic and spin-orbit terms as well as two-body, three-body, spinspin, spin-other-orbit and electrostatically correlated spinorbit interactions. As observed in table 6, their results are generally in very good agreement (within a few per cent) with our transition probabilities if we except the ${ }^{3} \mathrm{P}-{ }^{3} \mathrm{~F},{ }^{1} \mathrm{G}-{ }^{3} \mathrm{P}$ and ${ }^{1} \mathrm{D}-{ }^{3} \mathrm{~F}$ electric quadrupole lines for which larger discrepancies are observed.

\section{Conclusion}

Oscillator strengths and transition probabilities for allowed and forbidden lines in triply ionized praseaodymium have been obtained using a pseudo-relativistic Hartree-Fock model including core-polarization effects. Due to the lack of experimental data in this ion, the reliability of the new radiative parameters for electric dipole lines has been discussed and assessed through the excellent agreement observed between similar calculations and accurate laser lifetime measurements performed in the isoelectronic ion Ce III. In the case of forbidden lines, our results have been found to be in good agreement with theoretical data recently published if we except a few electric quadrupole transitions.

\section{Acknowledgments}

The authors acknowledge financial support from the Belgian National Fund for Scientific Research (FRS-FNRS) from which PQ is a Research Director. SEY also thanks the Marien Ngouabi University for its support.

\section{References}

Anisimova G P, Loginov A V and Tuchkin V I 2001 Opt. Spectrosc. 90315

Biémont É, Clar M, Enzonga Yoca S, Fivet V, Quinet P, Träbert E and Garnir H-P 2009 Can. J. Phys. 871275

Biémont É, Garnir H-P, Bastin T, Palmeri P, Quinet P, Li Z S, Zhang Z G, Lokhnygin V and Svanberg S 2001a Mon. Not. R. Astron. Soc. 321481

Biémont É, Garnir H-P, Palmeri P, Quinet P, Li Z S, Zhang Z G and Svanberg S 2001b Phys. Rev. A 64022503

Biémont É, Quinet P and Ryabchikova T A 2002 Mon. Not. R. Astron. Soc. 3361155

Cowan R D 1981 The Theory of Atomic Structure and Spectra (Berkeley, CA: University of California Press)

Crosswhite H M, Dieke G H and Carter W J 1965 J. Chem. Phys. 432047

Dodson C M and Zia R 2012 Phys. Rev. B 86125102

Dossing A 2005 Eur. J. Inorg. Chem. 20051425

Dzuba V A, Safronova U I and Johnson W R 2003 Phys. Rev. A $\mathbf{6 8} 032503$

Fraga S, Karwowski J and Saxena K M S 1976 Handbook of Atomic Data (Amsterdam: Elsevier)

Glushkov A V 1992 Opt. Spectrosc. 72293

Goldschmidt Z B 1968 Thesis Hebrew University of Jerusalem, Israel

Goldschmidt Z B, Pasternak A and Goldschmidt Z H 1968 Phys. Lett. A 28265

Hasegawa Y, Wada Y and Yanagida S 2004 J. Photochem. Photobiol. C 5183

Hemmila I 1995 J. Alloys Comp. 225480

Kramida A, Ralchenko Y and Reader J 2012 (NIST ASD Team) NIST Atomic Spectra Database (ver. 5.0) (Gaithersburg, MD: NIST) (http://physics.nist.gov/asd)

Li Z S, Lundberg H, Wahlgren G M and Sikström C M 2000 Phys. Rev. A 62032505

Li Z S et al 2001 J. Phys. B: At. Mol. Opt. Phys. 341349

Loginov A V and Tuchkin V I 2001 Opt. Spectrosc. 90631

Martin W C, Zalubas R and Hagan L 1978 Atomic Energy Levels-The Rare Earth Elements (NSRDS-NBS vol 60) (Washington DC: National Bureau of Standards)

Meftah A, Wyart J-F, Champion N and Tchang-Brillet W-Ü L 2007 Eur. Phys. J. D 4435

Migdalek J and Baylis W E 1979 J. Quant. Spectrosc. Radiat. Transfer 22127 
Migdalek J and Wyrozumska M 1987 J. Quant. Spectrosc. Radiat. Transfer 37581

Pasternak A 1970 Thesis Hebrew University of Jerusalem, Israel

Quinet P, Palmeri P, Biémont É, McCurdy M M, Rieger G, Pinnington E H, Wickliffe M E and Lawler J E 1999 Mon. Not. R. Astron. Soc. 307934

Savukov I M, Johnson W R, Safronova U I and Safronova M S 2003 Phys. Rev. A 67042504

Sen K D and Puri A 1989 Chem. Phys. Lett. 156505

Stanek M and Migdalek J 2004 J. Phys. B: At. Mol. Opt. Phys. 372707

Sugar J 1965 J. Opt. Soc. Am. 551058

Sugar J 1971a J. Opt. Soc. Am. 61727

Sugar J 1971b unpublished calculations

Wyart J-F, Blaise J and Worden E F 2005 J. Solid State Chem. 178589
Wyart J-F, Meftah A, Bachelier A, Sinzelle J, Tchang-Brillet W-Ü L, Champion N, Spector N and Sugar J 2006 J. Phys. B: At. Mol. Opt. Phys. 39 L77

Wyart J-F, Meftah A, Sinzelle J, Tchang-Brillet W-Ü L, Spector N and Judd B R 2008 J. Phys. B: At. Mol. Opt. Phys. 41085001

Wyart J-F, Meftah A, Tchang-Brillet W-Ü L, Champion N, Lamrous O, Spector N and Sugar J 2007 J. Phys. B: At. Mol. Opt. Phys. 403957

Wyart J-F, Tchang-Brillet W-Ü L, Spector N, Palmeri P, Quinet P and Biémont É 2001 Phys. Scr. 63113

Wybourne B G 2004 J. Alloys Compounds $\mathbf{3 8 0} 96$

Zhang Z G, Li Z S, Svanberg S, Palmeri P, Quinet P and Biémont É 2001a Eur. Phys. J. D 15301

Zhang Z G, Svanberg S, Jiang Z, Palmeri P, Quinet P and Biémont É 2001b Phys. Scr. 63122

Zhang Z G, Svanberg S, Quinet P, Palmeri P and Biémont É 2001c Phys. Rev. Lett. 87273001 